\title{
Additional Materials
}

For lecturers and research students using this book, the following supporting data are available:

- SC and SNC firm sample (used throughout the book)

- SC (RIEM) and SNC (RIEM) firm sample (used in Section 6.3)

For more information and to download the sample data please visit the following page: https://www.degruyter.com/document/isbn/9783110713664/html 